\title{
Archaeal communities in the deep-sea sediments of the South China Sea revealed by Illumina high-throughput sequencing
}

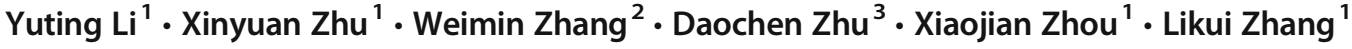 \\ Received: 7 February 2019 / Accepted: 22 April 2019 / Published online: 9 May 2019 \\ (C) Università degli studi di Milano 2019
}

\begin{abstract}
Purpose Archaea have important roles in global biogeochemical circulation. Although archaeal diversity and their ecological significance in deep-sea environments in the South China Sea (SCS) have been investigated, archaeal communities in deep-sea sediments below $2000 \mathrm{~m}$ water depth in the SCS are not well documented. The objective of our work was to investigate archaeal community structure in the four sediments (named as SCS2, SCS5, SCS8, and SCS10) collected from the SCS below $2000 \mathrm{~m}$ water depth.

Methods Illumina high-throughput sequencing was employed to reveal archaeal community structure. Archaeal communities were evaluated with QIIM software.

Result Archaeal communities in the four sediments were dominated by Thaumarchaeota (55\%), Bathyarchaeota (24\%), Woesearchaeota (6\%), Nanohaloarchaeota (4\%), and Euryarchaeota (3\%). Thaumarchaeota were abundant in the four samples. However, in SCS10, this phylum was almost exclusively represented. We revealed for the first time the presence of Nanohaloarchaeota in SCS2, SCS5, and SCS8. Comparative analysis showed that (1) the archaeal communities varied between the samples and (2) the samples varied between the samples. The detected archaea in each sample are known to be potentially participating in the carbon, nitrogen, and sulfur cycles, and methane metabolism.

Conclusion We present a comparative picture of archaeal communities, augmenting the current knowledge on archaeal diversity in deep-sea sediment environments in the SCS.
\end{abstract}

Keywords Archaeal diversity $\cdot$ Deep-sea sediment $\cdot$ High-throughput sequencing $\cdot$ Ecological function $\cdot 16 \mathrm{~S}$ rDNA

Weimin Zhang

wmzhang@gdim.cn

Daochen Zhu

dczhucn@hotmail.com

Likui Zhang

lkzhang@yzu.edu.cn

1 Marine Science \& Technology Institute, Department of Environmental Science and Engineering, Yangzhou University, No. 196 Huayang West Road, Hanjiang District, Yangzhou, Jiangsu, China

2 State Key Laboratory of Applied Microbiology Southern China, Guangdong Provincial Key Laboratory of Microbial Culture Collection and Application, Guangdong Open Laboratory $\backslash$ of Applied Microbiology, Guangdong Institute of Microbiology, Guangzhou, China

3 School of Environmental and Safety Engineering, Jiangsu University, Zhenjiang, Jiangsu, China

\section{Introduction}

As the third domain of life, archaea are thought to play important roles in global biogeochemical processes (Cavicchioli 2011). Early studies showed that archaea inhabit extreme environments. However, increasing evidence from culture independent technologies showed that archaea are ubiquitous in non-extreme environments (Cavicchioli 2011). Currently, archaea have been classified into two proposed super phyla with several new phylum-level lineages: "TACK" (Thaumarchaeota, Aigarchaeota, Crenarchaeota, Korarchaeota, Bathyarchaeota, and Lokiarchaeota) and "DPANN" (Diapherotrites, Parvarchaeota, Aenigmarchaeota, Nanoarchaeota, Nanohaloarchaeota, Pacearchaeota, Woesearchaeota, and Micrarchaeota) (Eme and Doolittle 2015). Recent studies on metagenomics and single-cell genomics suggest that archaea have versatile metabolic functions (Offre et al. 2013). Widespread presence of archaea in various environments and their 
versatile metabolisms indicate their important contribution in the global biogeochemical cycles of carbon, nitrogen, and sulfur.

Archaea are widely distributed in deep-sea sediments, where they display a remarkable diversity (Schleper et al. 2005; Brochier-Armanet et al. 2011). As an important component in some sedimentary ecosystems, archaea contribute to a large fraction of the biomass and their abundance is similar to that of bacteria in marine sediments (Lipp et al. 2008; Lloyd et al. 2013). Despite their high abundance, the metabolism of most archaea remains unknown due to the lack of cultivated representative species in the laboratory.

As the largest marginal sea $\left(3.5 \times 10^{6} \mathrm{~km}^{2}\right)$ in the Western Pacific Ocean, the South China Sea (SCS) is characterized by a huge oligotrophic subtropical and tropical water body with usually undetectable nitrate and phosphate in the euphotic zone (Moisander et al. 2008). The typical ecosystem in the SCS provides a comfortable habitat for microbial growth. Recently, archaeal communities in the sediments from various regions of the SCS have been frequently investigated (Jiang et al. 2007; Liao et al. 2009; Wang et al. 2010; Hu et al. 2011; Xia et al. 2015; Liu et al. 2017; Yu et al. 2017), reporting that Thaumarchaeota, Bathyarchaeota, Woesearchaeota, and Euryarchaeota are the dominant archaeal phyla. However, most of the investigated sediments were collected from the SCS less than or close to $2000 \mathrm{~m}$ depth. Currently, knowledge on archaeal communities in the SCS below $2000 \mathrm{~m}$ depth is limited.

Here, for the first time, we examined archaeal communities and their abundance in the four sediments collected from the SCS below $2000 \mathrm{~m}$ water depth by Illumina high-throughput sequencing. The primary goal of this study was to establish the archaeal communities of each sample. For this purpose, we sequenced the hypervariable V4 region of archaeal 16S rRNA gene. Our results showed that the diversity of archaea in the four sediments covered 10 archaeal phyla, 9 classes, 4 orders, 4 families, 2 genera, and 1 species, thus increasing the archaeal communities in deep-sea sediments in the SCS. Archaeal communities in the four sediments were dominated by the order of Thaumarchaeota (55\%), Bathyarchaeota (24\%), Woesearchaeota (6\%), Nanohaloarchaeota (4\%), and Euryarchaeota (3\%). Thaumarchaeota were abundantly ubiquitous in each sample; however, Thaumarchaeota were the only archaeal phylum in SCS10. To the best of our knowledge, this was the first report to reveal the presence of Nanohaloarchaeota in the deep-sea sediments in the SCS. The detected archaea in each sample are known to potentially participate in the carbon, nitrogen, and sulfur cycles, and methane metabolism.

\section{Materials and methods}

\section{Sample collection}

The four deep-sea sediments in this study were collected from the SCS at water depths ranging from 2026.6 to $2448.3 \mathrm{~m}$ as described by Zhu et al. (2013). The sampling sites are shown in Fig. 1. After sediment collection, samples were transferred to sterilized plastic tubes and frozen at $-80{ }^{\circ} \mathrm{C}$ before being processed.

\section{DNA extraction and PCR amplification}

Total metagenomic DNA from the four sediments was extracted from $0.25 \mathrm{~g}$ (wet weight) by using a Mo Bio soil DNA extraction kit (Carlsbad, CA, USA) according to manufacturer's instructions. Total DNA was eluted in $50 \mu \mathrm{L}$ elution buffer supplied with this kit. The concentrations of the DNA extracted were determined with a Nanodrop 2000 (Thermo Fisher Scientific, MA, USA) and its quality was analyzed by running a $0.8 \%$ agarose gel.

All PCR reactions were performed in $50 \mu \mathrm{L}$ reaction mixtures, consisting of $25 \mu \mathrm{L}$ of Phusion ${ }^{\circledR}$ High-Fidelity PCR Master Mix (New England Biolabs, MA, USA), $0.2 \mu \mathrm{M}$ of the 16S rRNA universal primers, and $10 \mathrm{ng}$ DNA. The universal primer set (U519F and 806R) was used to amplify the hypervariable V4 region of archaeal 16S rRNA gene, where the sequences of the forward and reverse primers were 5'CAG YMG CCR CGG KAA HAC C-3' and 5'-GGA CTA CNN GGG TAT CTA AT-3', respectively. Thermal cycling was initialized by denaturation at $98^{\circ} \mathrm{C}$ for $2 \mathrm{~min}$, followed by 30 cycles of denaturation at $98^{\circ} \mathrm{C}$ for $10 \mathrm{~s}$, annealing at $50^{\circ} \mathrm{C}$ for $30 \mathrm{~s}$, and elongation at $72{ }^{\circ} \mathrm{C}$ for $60 \mathrm{~s}$, with a final extension step at $72{ }^{\circ} \mathrm{C}$ for $5 \mathrm{~min}$. The PCR products were analyzed on a $2 \%$ agarose gel, and the amplified DNA ( $300 \mathrm{bp}$ ) was purified with GeneJET Gel Extrac-tion Kit (Thermo Fisher Scientific).

\section{Illumina high-throughput sequencing}

NEB Next ${ }^{\circledR}$ Ultra ${ }^{\mathrm{TM}}$ DNA Library Prep Kit was used to construct sequencing libraries. The library quality was assessed by the Qubit@2.0 Fluorometer (Thermo Fisher Scientific) and Agilent Bioanalyzer 2100 system. The libraries were sequenced (250 bp paired-end) on an Illumina MiSeq platform (New England Biolabs) 2500 at Novogene (Beijing, China): pairedend reads of 2500 and $250 \mathrm{bp}$ were generated by the addition of different multiple indexing barcodes, in accordance with the manufacturer's recommendations. Complete sequencing data were submitted to the NCBI Short Read Archive database under accession numbers: SRP137922 for SCS5, SRP137923 for SCS2, SRP137924 for SCS10, and SRP137925 for SCS8. 
Fig. 1 Map of sampling sites in the South China Sea

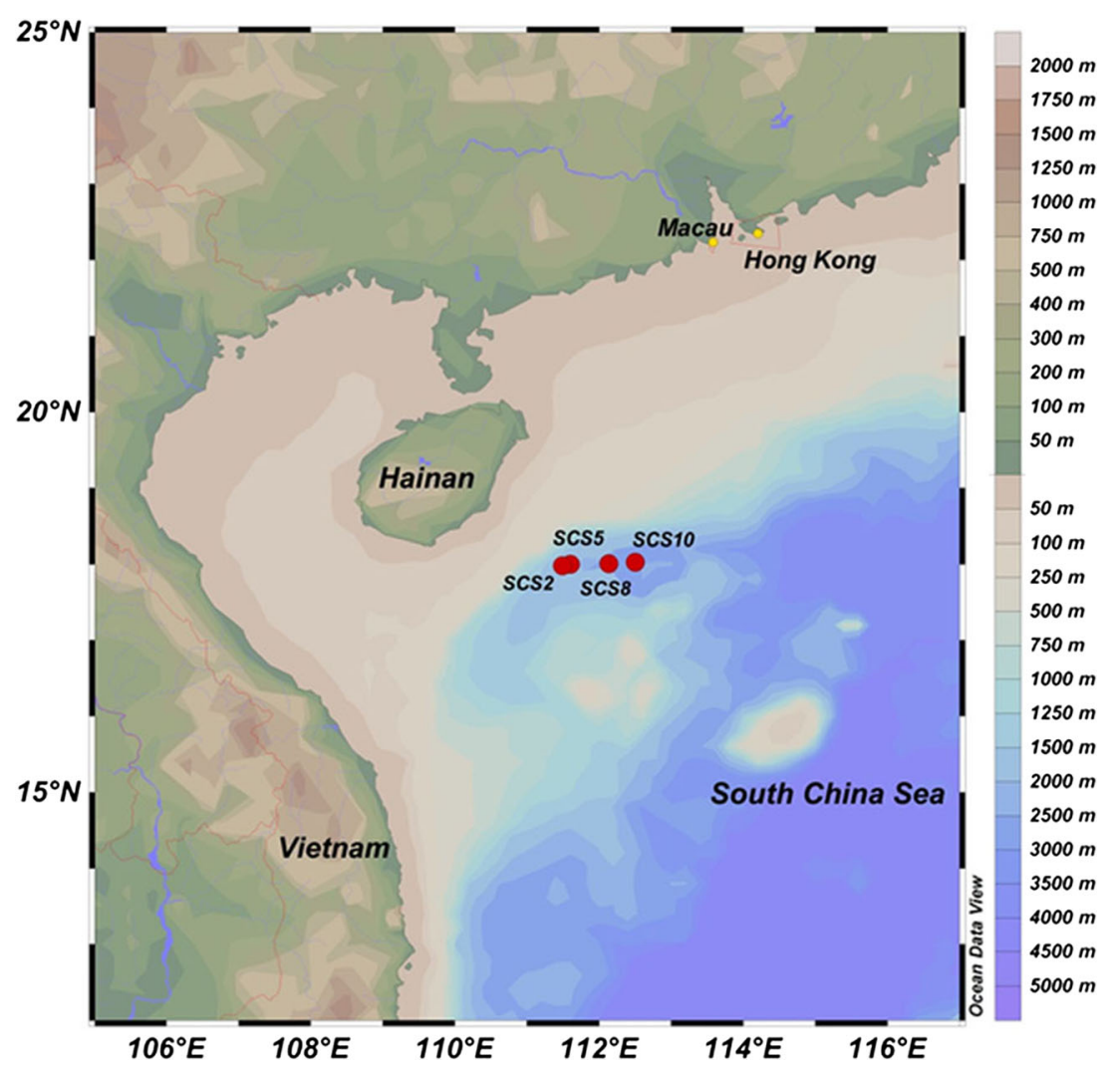

Based on barcode sequences, the reads from the original DNA fragments were merged using FLASH software. By means of QIIME (quantitative insights into microbial ecology) (Caporaso et al. 2010), the effective tags were generated (Haas et al. 2011). During construction of OTUs (operational taxonomic units), the effective tags at $\geq 97 \%$ similarity were clustered by UPARSE (Edgar 2013) and classified into one OTU. RDP classifier and GreenGene database were used to annotate a representative sequence of each OTU and its taxonomic information (DeSantis et al. 2006; Wang et al. 2007). For species analysis, the sequences with $\geq 97 \%$ similarity were assigned to the same OTU using UPARSE.

The most abundant 35 phyla of the archaeal species were selected and clustered, based on their abundance in each sample by QIIME, to construct a hierarchical clustering heat-map.

\section{Diversity analysis}

The QIIME software package was used to perform the analysis of alpha diversity (within samples) and beta diversity (among samples). In alpha diversity analysis, the OTUbased microbial community richness (Chao 1 estimator) and diversity (Shannon index) were calculated and rarefaction curves representing the amount of OTUs in each sample were generated. In beta diversity analysis, QIIME software package was used to perform cluster analysis and two dimensional principal coordinates analysis (PCoA); both weighted and unweighted UniFrac distances (Lozupone et al. 2011) were calculated to generate the index of beta diversity.

\section{Results}

\section{Sequencing data}

Sequencing information on total tags, effective tags, unique tags, diversity index, and estimators of richness is summarized in Table 1. The Illumina-based analysis of the hypervariable V4 region of the archaeal 16S rRNA gene produced 117,197 total tags. After filtering and removing potential erroneous sequences, a total of 109,641 effective tags and 7556 unique tags were obtained.

Based on $97 \%$ similarity, a total of 186 OTUs for archaeal diversity were obtained from the four samples, and the order of the OTU numbers ranging from high to low was 80 for SCS2, 73 for SCS8, 24 for SCS5, and 9 for SCS10 (Table 1). A Venn diagram was created, which showed that the four samples shared 6 OTUs (Fig. 2), suggesting the presence of similar archaeal groups in each sample. Furthermore, various numbers of shared and unique OTUs exist in the four samples, suggesting that the four sediments have different archaeal community structure, albeit harboring similar archaeal species. 
Table 1 Sequencing information in this study

\begin{tabular}{lllll}
\hline Sequencing information & SCS10 & SCS2 & SCS5 & SCS8 \\
\hline Number of total tags & 7197 & 51,914 & 6929 & 51,157 \\
Number of effective tags & 7145 & 48,767 & 4744 & 48,985 \\
Number of unique tags & 52 & 3147 & 2185 & 2172 \\
OTUs (97\% similarity) & 9 & 80 & 24 & 73 \\
Shannon index & 1.061 & 4.139 & 2.268 & 4.255 \\
Chao 1 index & 8 & 70.25 & 18.25 & 54 \\
\hline
\end{tabular}

The Shannon's diversity index reported the same order for archaeal diversity, i.e., SCS8 (Shannon $=4.225)$, SCS2 $($ Shannon $=4.139)$, SCS5 $($ Shannon $=2.268)$, and SCS10 (Shannon $=1.061)$. All rarefaction curves approached an asymptote (Fig. 3), indicating that the sampling depth was sufficient to capture the whole archaeal communities in each sample.

\section{Archaeal community analysis}

As shown in Table 2, a total of 386,015 effective tags were created in all the four sediments, which covered 10 phyla, 9 classes, 4 orders, 4 families, 2 genera, and 1 species (Table 2).

At the phylum level, Thaumarchaeota (100\%) were found in SCS10, suggesting that Thaumarchaeota were an almost unique archaeal phylum in this sample (Fig. 4). In addition, Thaumarchaeota (48\%), Bathyarchaeota (37\%), Euryarchaeota (5\%), Aigarchaeota (2\%), and Woesearchaeota (2\%) were the dominant archaeal phyla in SCS2 (Fig. 4). Furthermore, SCS2 had relatively low abundance of Nanohaloarchaeota, MHVG (marine hydrothermal vent group), SM1K20, and other unknown archaea $(<1 \%)$. It is noteworthy that SM1K20 was only detected in SCS2, despite its abundance being low.

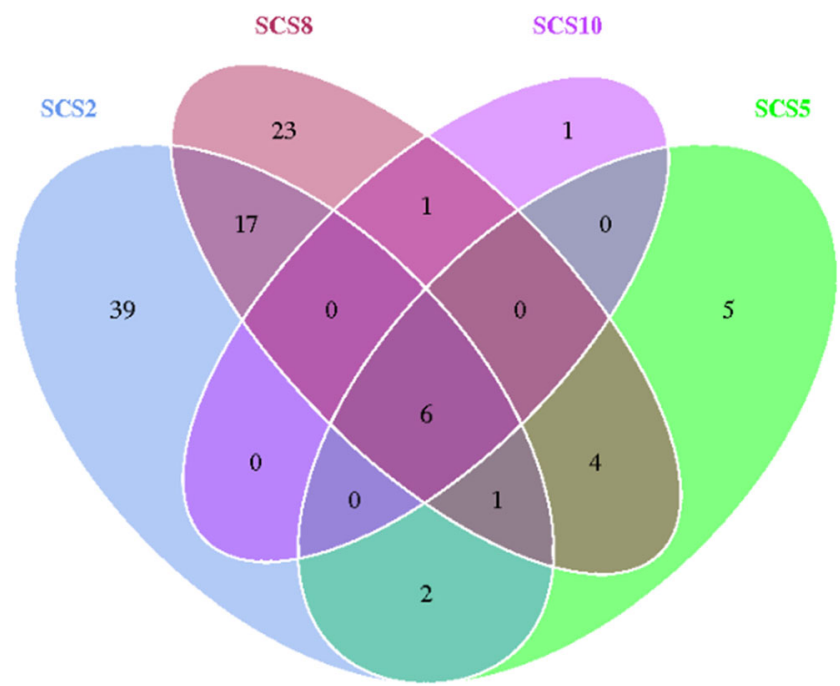

Fig. 2 Venn diagrams of the OTUs for archaeal communities in each sample. Shared and unique OTUs in each sample were calculated based on $97 \%$ similarity. The numbers of OTUs are indicated inside the diagram
In SCS5, 44\% of sequences at the phylum level were affiliated with the Nanohaloarchaeota phylum. However, SCS2 and SCS8 had low abundance of this phylum, while in SCS10, no sequence of this phylum was detected. In contrast, Nanohaloarchaeota are a major phylum in SCS5. This is the first report on the presence of Nanohaloarchaeota in deep-sea sediments in the SCS. In addition to Nanohaloarchaeota, Woesearchaeota (34\%) and Thaumarchaeota (22\%) were also detected in SCS5. Furthermore, Bathyarchaeota were estimated to be only $0.08 \%$ in SCS5, which is in sharp contrast to SCS2 (37\%). In addition, SCS5 harbored low abundance of Euryarchaeota and other unknown archaea. Thus, the archaeal communities in SCS5 mostly consisted of Nanohaloarchaeota, Woesearchaeota, and Thaumarchaeota.

As observed in SCS10, SCS2, and SCS5, 59\% of sequences at the phylum level belong to Thaumarchaeota phylum in SCS8, suggesting that Thaumarchaeota are widespread in all the four samples and their abundance is relatively high. Besides, $17 \%, 8 \%, 7 \%, 4 \%, 2 \%$, and $1 \%$ of sequences in SCS8 originated from Bathyarchaeota, Woesearchaeota, other unknown archaea, Nanohaloarchaeota, MHVG, and Euryarchaeota, respectively. Furthermore, Diapherotrites and Aenigmarchaeota were exclusively present in SCS8. Overall, SCS8 was found to harbor more archaeal species than SCS2, SCS5, and SCS10.

Based on the annotated archaeal species and their relative abundance, Nanohaloarchaeota, Woesearchaeota, Thaumarchaeota, SM1K20, Aigarchaeota, Euryarchaeota, Bathyarchaeota, Aigarchaeota, and Diapherotrites were detected in all four sediments. The 35 most abundant phyla in all four samples were analyzed by hierarchically clustering heat mapping, suggesting that the cluster of most phyla varied despite their occurrence in the four samples (Fig. 5). Specifically, SM1K20, Aigarchaeota, Euryarchaeota, and Bathyarchaeota were mostly clustered in SCS2, Nanohaloarchaeota and Woesearchaeota in SCS5, Aigarchaeota and Diapherotrites in SCS8, and Thaumarchaeota in SCS10.

At the class level, 9 classes were identified to affiliate with Thaumarchaeota, Euryarchaeota, Aigarchaeota, and Aenigmarchaeota in all four sediments. As the predominant archaeal phylum, the detected Thaumarchaeota covered 5 classes: MBG (marine benthic group) B, Group C3, SCG, AK8, and MBG A, among which MBG B was the most 
Fig. 3 Rarefaction curves of $16 \mathrm{~S}$ rDNA sequences of archaeal communities in each sample. Rarefaction curves were generated based on OTUs at $97 \%$ similarity

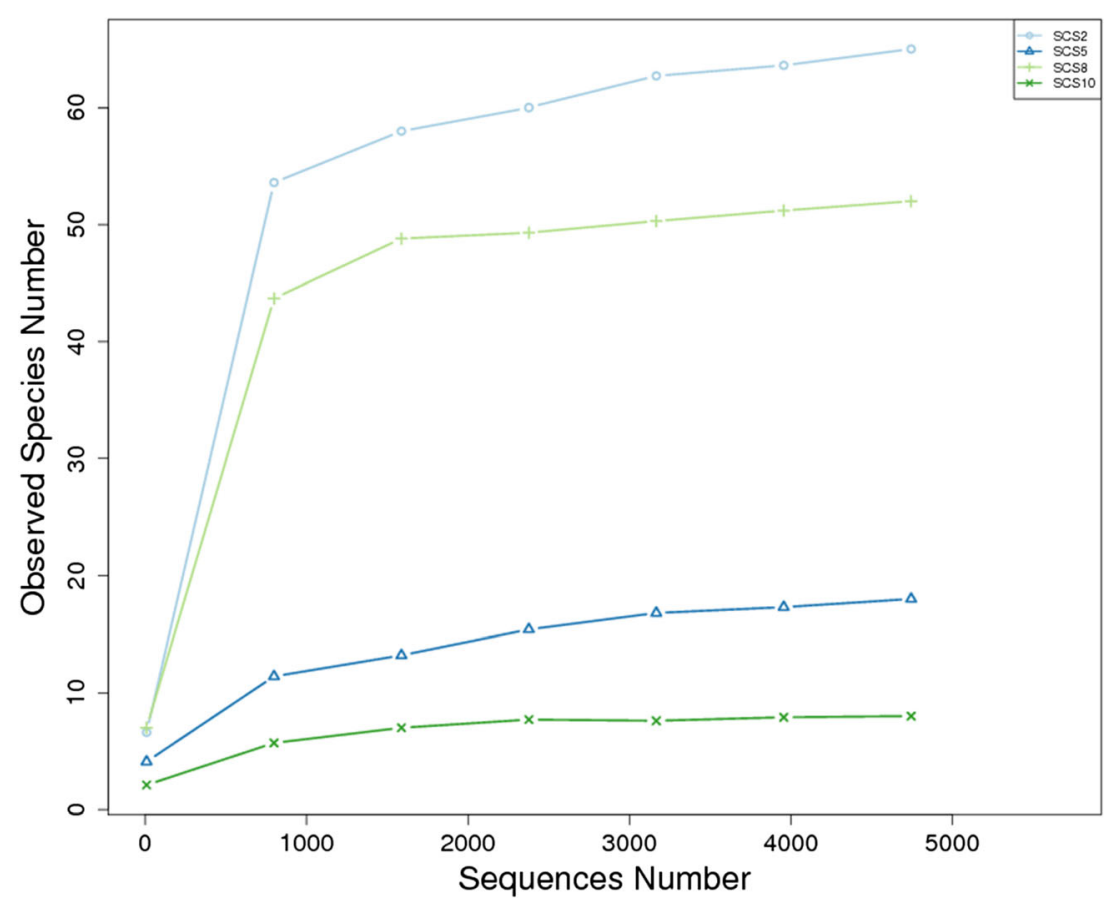

abundant (40\%) in SCS2, and was followed by Group C3, SCG, AK8, and MBG A (Table 2). In addition, Thermoplasmata and Methanomicrobia in Euryarchaeota phylum were the two identified classes in this study.

Table 2 Archaeal community composition in each sample at the class, order, family, genus, and species levels

\begin{tabular}{|c|c|c|c|c|}
\hline \multirow[t]{2}{*}{ Sequencing information } & \multicolumn{4}{|c|}{ Relative abundance (\%) } \\
\hline & SCS10 & $\mathrm{SCS} 2$ & SCS5 & SCS8 \\
\hline \multicolumn{5}{|l|}{ Class } \\
\hline MBG B & ND & 39.84 & 0.10 & 20.89 \\
\hline Group C3 & ND & 5.65 & ND & 1.43 \\
\hline SCG & ND & ND & ND & 1.12 \\
\hline AK8 & ND & 0.53 & ND & ND \\
\hline MBG A & ND & ND & 0.02 & 0.19 \\
\hline Thermoplasmata & ND & 4.79 & 0.06 & 1.20 \\
\hline Methanomicrobia & ND & 0 & 0.19 & ND \\
\hline THSCG & ND & 2.21 & ND & ND \\
\hline DSEG & ND & ND & ND & 0.13 \\
\hline \multicolumn{5}{|l|}{ Order } \\
\hline Thermoplasmatales & ND & 3.48 & ND & 0.82 \\
\hline $19 \mathrm{c} 33$ & ND & 1.31 & ND & ND \\
\hline MBG E & ND & ND & 0.06 & 0.38 \\
\hline Methanosarcinales & ND & ND & 0.19 & ND \\
\hline \multicolumn{5}{|l|}{ Family } \\
\hline MBG D & ND & 3.37 & ND & ND \\
\hline ANT06-05 & ND & 0.06 & ND & 0.72 \\
\hline Methanosarcinaceae & ND & ND & 0.19 & ND \\
\hline CCA47 & ND & 0.04 & ND & ND \\
\hline \multicolumn{5}{|l|}{ Genus } \\
\hline Candidatus Nitrosopumilus & 40.79 & 0.99 & 14.63 & 11.45 \\
\hline Methanomethylovorans & ND & ND & 0.19 & ND \\
\hline \multicolumn{5}{|l|}{ Species } \\
\hline Archaeon GW2011 AR13 & ND & 0.02 & ND & 0.02 \\
\hline
\end{tabular}

ND, not detected
Thermoplasmata was present in SCS2, SCS8, and SCS5, while Methanomicrobia was exclusively found in SCS5. Furthermore, THSCG (terrestrial hot spring crenarchaeota group) belonging to the phylum Aigarchaeota was only detected in SCS2 with $2 \%$ of relative abundance, and DSEG (deep-sea euryarchaeotal group), which is affiliated with the phylum Aenigmarchaeota, was observed exclusively in SCS8 with $0.13 \%$ of relative abundance.

At the order level, 4 orders were detected in all four sediments with low abundance: Thermoplasmatales, 19c-33, MBGE, and Methanosarcinales (Table 2), which are members of Euryarchaeota. Thermoplasmatales were present in SCS2 and SCS8 while 19c-33 was found exclusively in SCS2. In addition, MBG E was found in SCS5 and SCS8 and Methanosarcinales were observed exclusively in SCS5.

At the family level, 4 families were observed in all four sediments with low abundance: MBGD, CCA47, ANT06-05, and Methanosarcinaceae (Table 2). As observed in the order level, the four families belong to Euryarchaeota. MBG D and CCA47 were only present in SCS2, and Methanosarcinaceae was only observed in SCS5. Furthermore, ANT06-05 was found in SCS2 and SCS8.

At the genus level, two genera were detected in the four sediments: Candidatus Nitrosopumilus and Methanomethylovorans, which were affiliated with Thaumarchaeota and Euryarchaeota, respectively (Table 2). The most abundant archaeal genus was Candidatus Nitrosopumilus, which was ubiquitous, with a relative abundance of $41 \%, 1 \%, 15 \%$, and $11 \%$ in SCS10, SCS2, SCS5, and SCS8, respectively. By contrast, Methanomethylovorans were exclusively observed in SCS5 at a low abundance. 
Fig. 4 Relative abundance of archaeal communities at the phylum level. The percentage of each phylum in each sample is indicated with each color

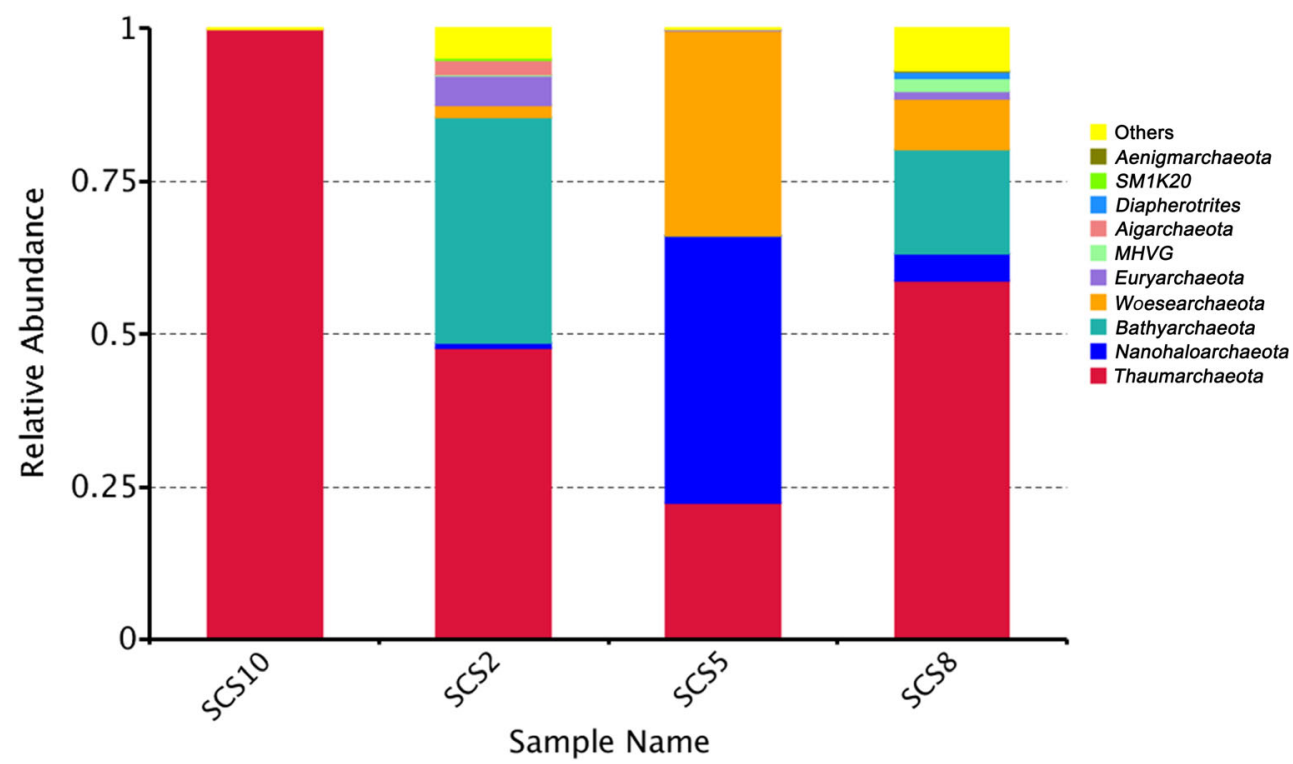

Most archaea were not identified at the species level. However, one archaeon GW2011AR13, which is a member of the Woesearchaeota, was detected in both SCS2 and SCS8 at the same relative low abundance $(0.02 \%)$ (Table 2$)$.

\section{Beta diversity analysis of the four samples}

Based on the weighted UniFrac distance and unweighted UniFrac distance cluster analysis, dissimilarity coefficients between two samples were measured for all the four samples to estimate the divergence of archaeal species between them. Lower dissimilarity coefficients suggest a lower divergence of microbial species. In this study, we found that the lowest dissimilarity coefficient was between SCS2 and SCS8 (0.281) and the highest between SCS5 and SCS10 (0.741) (Fig. 6).

Based on unweighted UniFrac PCoA, where the archaeal abundance was not considered, the archaeal communities of SCS2, SCS5, SCS8, and SCS10 were separated from each other according to PC1 and PC2 (55\% and 27\% explained variance, respectively) (Fig. 7a), suggesting that archaeal diversity varied in each sample. Furthermore, SCS10 had a relatively long distance from SCS2, SCS5, and SCS10, while the distances among SCS2, SCS5, and SCS8 were relatively close together, indicating that SCS10 has clearly distinct archaeal communities from SCS2, SCS5, and SCS10.

Based on weighted UniFrac PCoA, where the archaeal abundance was considered, SCS2, SCS5, SCS8, and SCS10 had long distances between each other according to $\mathrm{PC} 1$ and PC2 (63\% and $31 \%$ explained variance, respectively) (Fig. 7b), suggesting the existence of clear divergence in archaeal diversity in each sample. Furthermore, the distance between SCS2 and SCS8 was closer than that between SCS5 and SCS10, suggesting that archaeal communities between SCS2 and SCS8 are more similar than those between SCS5 and SCS10. These results were confirmed by analysis of beta diversity index as described above.

\section{Discussion}

In this work, we used a barcoded Illumina high-throughput sequencing platform to investigate archaeal communities in

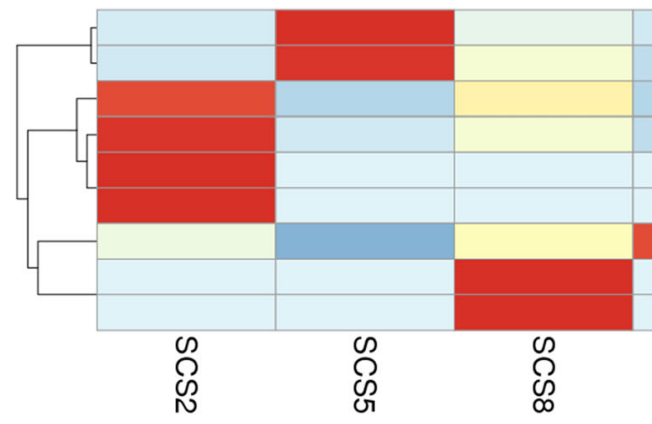

Fig. 5 Hierarchical clustering heat-map of archaeal communities in each sample. The neighbor-joining method was used to reconstruct the phylogenetic tree. The relative percentage of each phylum within each

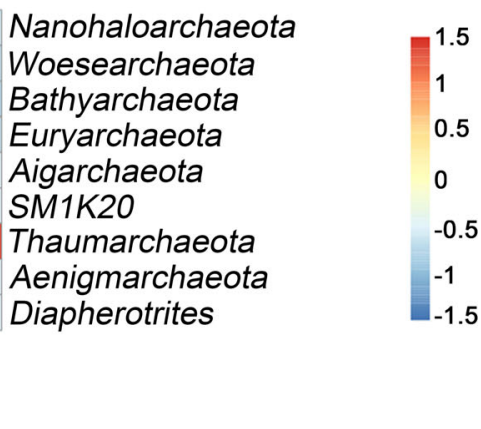

sample (vertical clustering) or among four samples (horizontal clustering) is depicted in the heat-map. Color intensity indicated at the right of the figure represents the relative values for each phylum 
Fig. 6 Beta diversity index of the archaeal communities in the four samples. Beta diversity indices were measured based on weighted UniFrac distance. The numbers in the grid represent the weighted UniFrac distances

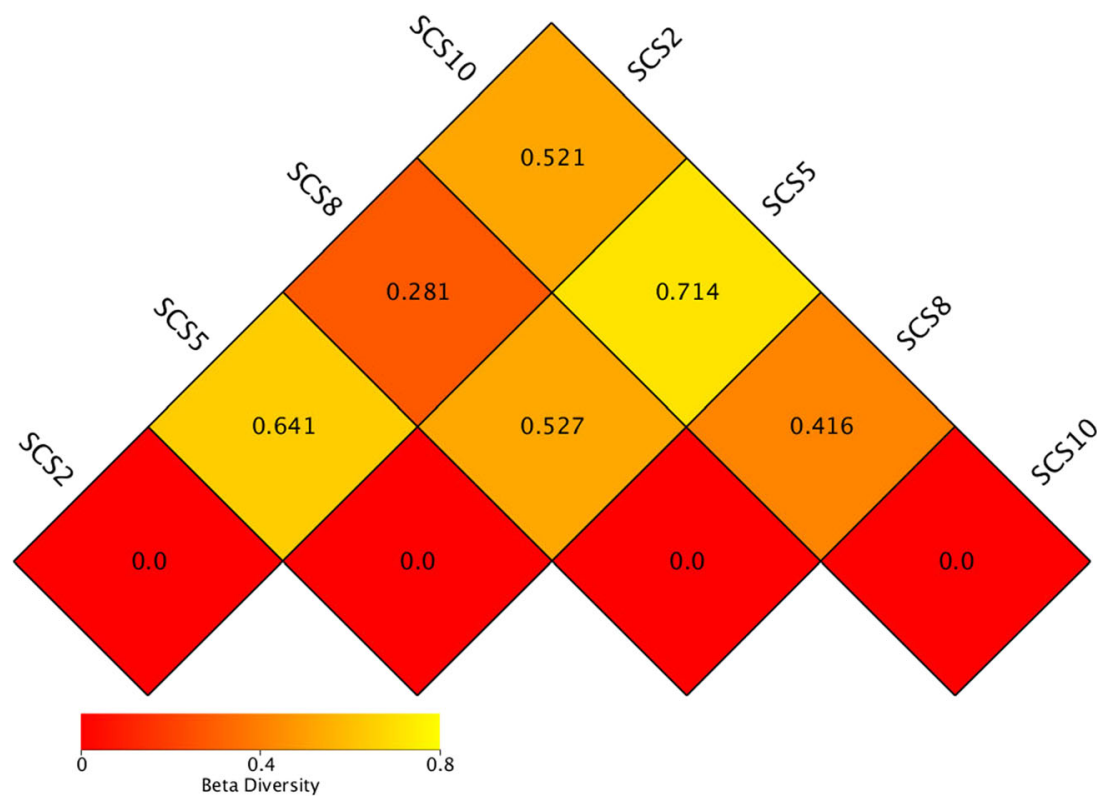

sediments from the SCS below $2000 \mathrm{~m}$ water depth, and detected 10 phyla common to all samples, which most abundant were Thaumarchaeota (55\%), Bathyarchaeota (24\%), Woesearchaeota (6\%), Nanohaloarchaeota (4\%), and Euryarchaeota $(3 \%)$. In addition, other five phyla were detected at lower abundance: MHVG (1\%), Aigarchaeota (1\%), Diapherotrites $(0.5 \%)$, SM $1 \mathrm{~K} 20(0.2 \%)$, and Aenigmarchaeota $(0.1 \%)$. Overall, the deep-sea sediments below $2000 \mathrm{~m}$ water depth in the SCS harbor abundant archaeal communities.

Thaumarchaeota were the most dominant phylum since they were detected in each sample at abundances ranging from 22 to $100 \%$. Other studies have also detected Thaumarchaeota in deep-sea sediments of the SCS (Wang et al. 2010; Liu et al. 2017), where they were found to possess the dissimilatory ammonia oxidation pathway for generating energy by ammonia oxidation (Stahl and de la Torre 2012). Taken together, the abundant Thaumarchaeota might be involved in the effective removal of ammonia in deep-sea sediments of the SCS.

The Thaumarchaeota detected in this work comprised 5 classes: MBG B, Group C3, SCG, AK8, and MBGA, among which MBG B was the most abundant class. MBG $B$ is known to participate in sulfate-reduction and $\mathrm{CH}_{4}$ oxidation (Wang et al. 2010). As a ubiquitous archaeal group in oceanic sediments, Group C3 was also found to be abundantly present in SCS2, SCS5, and SCS8. Previous studies revealed that Group $\mathrm{C} 3$ is involved in the sulfur and carbon cycle (Na et al. 2015). Overall, the detected MBG B and Group C3 in the deep-sea sediments in the SCS might contribute to $\mathrm{CH}_{4}$ and acetate metabolism.

Bathyarchaeota were the second most abundant phylum to be detected in SCS2, SCS5, and SCS8 samples. Metagenome and single-cell sequencing revealed that Bathyarchaeota possibly participate in the degradation of detrital proteins and in acetogenesis (He et al. 2016; Lazar et al. 2016). Furthermore, Bathyarchaeota harbor the complete $\mathrm{CH}_{4}$ metabolism pathway, suggesting that they might play an important role in $\mathrm{CH}_{4}$ metabolism (Evans et al. 2015). Previous studies have shown that Bathyarchaeota frequently inhabit various regions of SCS (Jiang et al. 2007; Wang et al. 2010; Jiang et al. 2011; Zhang et al. 2012; Chen et al. 2013). The current results are congruent with previous finding and support an important role in carbon cycle and $\mathrm{CH}_{4}$ metabolism for Bathyarchaeota in the SCS.

Woesearchaeota were initially grouped into DHVEG-6 branch of Euryarchaeota, and at present belong to DPANN superphylum (Eme and Doolittle 2015). Woesearchaeota are thought to be haloarchaea, and have a close relationship lineage with Halobacteriaceae (Han et al. 2017); however, their ecological function remains unknown. Similar to previous studies on archaeal community in the SCS (Wang et al. 2010; Liu et al. 2017), we also detected the presence of Woesearchaeota in SCS2, SCS5, and SCS8 samples. Our observations indicate that Woesearchaeota are widespread in the deep-sea sediments in the SCS.

Currently, Nanohaloarchaeota have been detected in only hypersaline soils and hypersaline sediments (Di Meglio et al. 2016; Han et al. 2017; Mora-Ruiz et al. 2018). Due to lack of cultivated strain, the ecological function of Nanohaloarchaeota remains unclear. In this study, we revealed the presence of Nanohaloarchaeota in SCS2, SCS5, and SCS8 samples. To our knowledge, this was the first report of Nanohaloarchaeota with a notable abundance in deep-sea sediments in the SCS, increasing the existing knowledge regarding the archaeal community structure in deep-sea sediments of the SCS. 

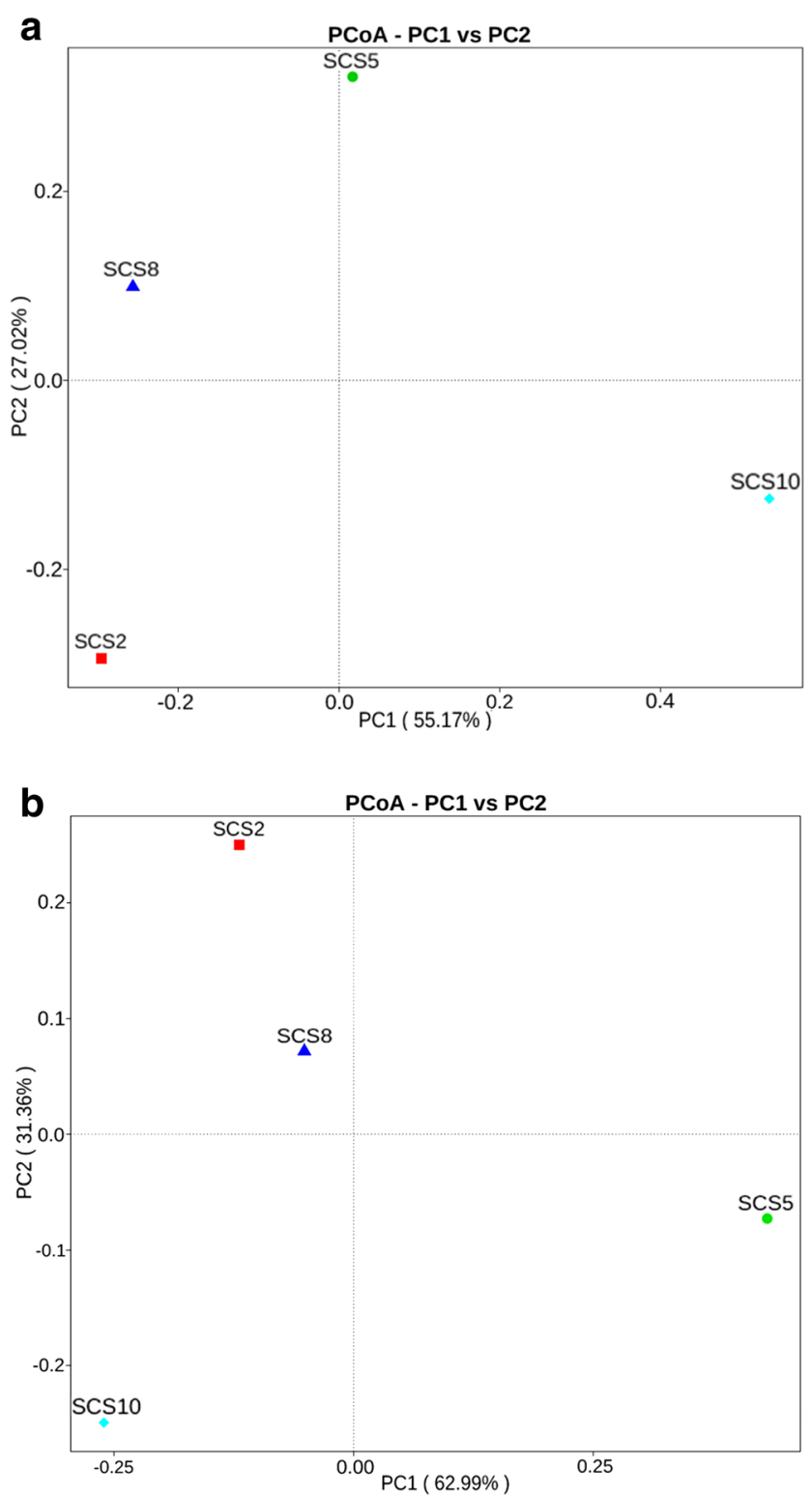

Fig. 7 Principal coordinate analysis (PCoA) of each sample. a PCoA plots are based on unweighted UniFrac metrics for each sample. b PCoA plots are based on weighted UniFrac metrics for each sample

Most annotated sequences of Euryarchaeota belong to Thermoplasmata and few sequences are Methanomicrobia. Species within the archaeal class Thermoplasmata are thought to participate in methanogenic activities (Iino et al. 2013; Poulsen et al. 2013; Petersen et al. 2014). In this study, we detected the presence of Euryarchaeota containing Thermoplasmata and Methanomicrobia, and the abundance of Thermoplasmata were higher than that of Methanomicrobia, suggesting their potential roles in methanogenesis. Previous studies on archaeal diversity have shown that Thermoplasmata and Methanomicrobia are distributed in the deep-sea sediments in the SCS (Wang et al. 2010; Liu et al. 2017; Niu et al. 2017). Taken together, these results suggest that Euryarchaeota is one of the important members of archaea in the deepsea environments of the SCS, which might participate in $\mathrm{CH}_{4}$ metabolism.

Similar to previous reports on archaeal communities in the SCS (Wang et al. 2010; Liu et al. 2017), we also detected five other archaeal phyla at lower abundance: MHVG, Aigarchaeota, Diapherotrites, SM1K20, and Aenigmarchaeota. These observations suggest that these low abundance archaeal lineages are ubiquitous in deep-sea environments of the SCS.

In conclusion, we first reported the comparative analysis of archaeal communities in four deep-sea sediments from various regions of the SCS (below $2000 \mathrm{~m}$ water depth) using Illumina high-throughput sequencing. Our results demonstrated that archaeal communities in these samples were mostly composed of Thaumarchaeota, Bathyarchaeota, Nanohaloarchaeota, MCG, Woesearchaeota, and Euryarchaeota. Alpha diversity and beta diversity analysis showed that archaeal community composition and their abundance varied in each sediment of the SCS. Furthermore, we are the first to reveal the occurrence of Nanohaloarchaeota, augmenting the existing knowledge on the archaeal community structure in the deep-sea sediments of the SCS. Our results suggest that the archaeal communities detected could be involved in carbon, nitrogen, and sulfur cycles, as well as methane metabolism.

Acknowledgements The authors would like to thank Prof. Philippe Oge at Univ Lyon, INSA de Lyon, CNRS UMR 5240 for revising this manuscript, and Manyu Kang at Tongji University for drawing Fig. 1.

Funding This work was supported by the Academic Leader of Middle and Young People of Yangzhou University Grant to L.Z.; by the Practice Innovation Training Program for College Students in Jiangsu to Y. L (No. 201711117059Y); by the National Natural Science Foundation of China Grant (No. 41776156) to X.Z; by the Science and Technology Program of Guangzhou, China (No. 201607020018) and the Team Project of Natural Science Foundation of Guangdong Province (No. 2016A030312014) to W.Z.

\section{Compliance with ethical standards}

Conflict of interest The authors declare that they have no conflicts of interest.

Research involving human participants and/or animals N/A

Informed consent N/A 


\section{References}

Brochier-Armanet C, Forterre P, Gribaldo S (2011) Phylogeny and evolution of the archaea: one hundred genomes later. Curr Opin Microbiol 14:274-281

Caporaso JG, Kuczynski J, Stombaugh J, Bittinger K, Bushman FD, Costello EK, Fierer N, Pena AG, Goodrich JK, Gordon JI, Huttley GA, Kelley ST, Knights D, Koenig JE, Ley RE, Lozupone CA, McDonald D, Muegge BD, Pirrung M, Reeder J, Sevinsky JR, Turnbaugh PJ, Walters WA, Widmann J, Yatsunenko T, Zaneveld J, Knight R (2010) QIIME allows analysis of high-throughput community sequencing data. Nat Methods 7:335-336

Cavicchioli R (2011) Archaea - timeline of the third domain. Nat Rev Microbiol 9:51-61

Chen JQ, Wang FP, Jiang LJ, Yin XJ, Xiao X (2013) Stratified communities of active archaea in shallow sediments of the Pearl River estuary, Southern China. Curr Microbiol 67:41-50

DeSantis TZ, Hugenholtz P, Larsen N, Rojas M, Brodie EL, Keller $\mathrm{K}$, Huber T, Dalevi D, Hu P, Andersen GL (2006) Greengenes, a chimera-checked 16S rRNA gene database and workbench compatible with ARB. Appl Environ Microbiol 72:5069-5072

Di Meglio L, Santos F, Gomariz M, Almansa C, Lopez C, Anton J, Nercessian D (2016) Seasonal dynamics of extremely halophilic microbial communities in three Argentinian salterns. FEMS Microbiol Ecol 92

Edgar RC (2013) UPARSE: highly accurate OTU sequences from microbial amplicon reads. Nat Methods 10:996-998

Eme L, Doolittle WF (2015) Archaea. Curr Biol 25:R851-R855

Evans PN, Parks DH, Chadwick GL, Robbins SJ, Orphan VJ, Golding SD, Tyson (2015) Methane metabolism in the archaeal phylum Bathyarchaeota revealed by genome-centric metagenomics. Science 350: 434-438

Haas BJ, Gevers D, Earl AM, Feldgarden M, Ward DV, Giannoukos G, Ciulla D, Tabbaa D, Highlander SK, Sodergren E, Methé B, TZ DS, Consortium HM, Petrosino JF, Knight R, Birren BW (2011) Chimeric 16S rRNA sequence formation and detection in sanger and 454-pyrosequenced PCR amplicons. Genome Res 21:494-504

Han R, Zhang X, Liu J, Long QF, Chen LS, Liu DL, Zhu DR (2017) Microbial community structure and diversity within hypersaline Keke Salt Lake environments. Can J Microbiol 63:895908

He Y, Li M, Perumal V, Feng X, Fang J, Xie J, Sievert SM, Wang F (2016) Genomic and enzymatic evidence for acetogenesis among multiple lineages of the archaeal phylum Bathyarchaeota widespread in marine sediments. Nat Microbiol 1:16035

Hu AY, Jiao NZ, Zhang CLL (2011) Community structure and function of planktonic crenarchaeota: changes with depth in the South China Sea. Microbial Ecol 62:549-563

Iino T, Tamaki H, Tamazawa S, Ueno Y, Ohkuma M, Suzuki K, Igarashi Y, Haruta S (2013) Candidatus Methanogranum caenicola: a novel methanogen from the anaerobic digested sludge, and proposal of Methanomassiliicoccaceae fam. nov and Methanomassiliicoccales ord. nov., for a, methanogenic lineage of the class Thermoplasmata. Microb Environ 28:244-250

Jiang HC, Dong HL, Ji SS, Ye Y, Wu NY (2007) Microbial diversity in the deep marine sediments from the Qiongdongnan Basin in South China Sea. Geomicrobiol J 24:505-517

Jiang L, Zheng Y, Chen J, Xiao X, Wang F (2011) Stratification of archaeal communities in shallow sediments of the Pearl River
Estuary, Southern China. Antonie Van Leeuwenhoek 99:739751

Lazar CS, Baker BJ, Seitz K, Hyde AS, Dick GJ, Hinrichs KU, Teske AP (2016) Genomic evidence for distinct carbon substrate preferences and ecological niches of Bathyarchaeota in estuarine sediments. Environ Microbiol 18:1200-1211

Liao L, Xu XW, Wang CS, Zhang DS, Wu M (2009) Bacterial and archaeal communities in the surface sediment from the northern slope of the South China Sea. J Zhejiang Univ Sci B 10:890 901

Lipp JS, Morono Y, Inagaki F, Hinrichs KU (2008) Significant contribution of archaea to extant biomass in marine subsurface sediments. Nature 454:991-994

Liu HD, Zhang CLL, Yang CY, Chen SZ, Cao ZW, Zhang ZW, Tian JW (2017) Marine group II dominates planktonic archaea in water column of the Northeastern South China Sea. Front Microbiol 8

Lloyd KG, Schreiber L, Petersen DG, Kjeldsen KU, Lever MA, Steen AD, Stepanauskas R, Richter M, Kleindienst S, Lenk S, Schramm A, Jorgensen BB (2013) Predominant archaea in marine sediments degrade detrital proteins. Nature 496:215-218

Lozupone C, Lladser ME, Knights D, Stombaugh J, Knight R (2011) UniFrac: an effective distance metric for microbial community comparison. ISME J 5:169-172

Moisander PH, Beinart RA, Voss M, Zehr JP (2008) Diversity and abundance of diazotrophic microorganisms in the South China Sea during intermonsoon. ISME J 2:954-967

Mora-Ruiz MDR, Cifuentes A, Font-Verdera F, Perez-Fernandez C, Farias ME, Gonzalez B, Orfila A, Rossello-Mora R (2018) Biogeographical patterns of bacterial and archaeal communities from distant hypersaline environments. Syst Appl Microbiol 41: $139-150$

Na H, Lever MA, Kjeldsen KU, Schulz F, Jorgensen BB (2015) Uncultured Desulfobacteraceae and Crenarchaeotal group C3 incorporate C-13-acetate in coastal marine sediment. Env Microbiol Rep 7:614-622

Niu MY, Fan XB, Zhuang GC, Liang QY, Wang FP (2017) Methanemetabolizing microbial communities in sediments of the Haima cold seep area, northwest slope of the South China Sea. FEMS Microbiol Ecol 93

Offre P, Spang A, Schleper C (2013) Archaea in biogeochemical cycles. Annu Rev Microbiol 67:437-457

Petersen SO, Hojberg O, Poulsen M, Schwab C, Eriksen J (2014) Methanogenic community changes, and emissions of methane and other gases, during storage of acidified and untreated pig slurry. $\mathrm{J}$ Applied Microbiol 117:160-172

Poulsen M, Schwab C, Jensen BB, Engberg RM, Spang A, Canibe N, Hojberg O, Milinovich G, Fragner L, Schleper C, Weckwerth W, Lund P, Schramm A, Urich T (2013) Methylotrophic methanogenic Thermoplasmata implicated in reduced methane emissions from bovine rumen. Nat Commun $4: 1428$

Schleper C, Jurgens G, Jonuscheit M (2005) Genomic studies of uncultivated archaea. Nat Reviews Microbiol 3:479-488

Stahl DA, de la Torre JR (2012) Physiology and diversity of ammoniaoxidizing archaea. Annu Rev Microbiol 66:83-101

Wang P, Li T, Hu A, Wei Y, Guo W, Jiao N, Zhang C (2010) Community structure of archaea from deep-sea sediments of the South China Sea. Microb Ecol 60:796-806

Wang Q, Garrity GM, Tiedje JM, Cole JR (2007) Naive Bayesian classifier for rapid assignment of rRNA sequences into the new bacterial taxonomy. Appl Environ Microbiol 73:52615267 
Xia XM, Guo W, Liu HB (2015) Dynamics of the bacterial and archaeal communities in the northern South China Sea revealed by 454 pyrosequencing of the 16S rRNA gene. Deep-Sea Res Pt II117:97-107

Yu TT, Liang QY, Niu MY, Wang FP (2017) High occurrence of Bathyarchaeota (MCG) in the deep-sea sediments of South China Sea quantified using newly designed PCR primers. Env Microbiol Rep 9:374-382

Zhang Y, Su X, Chen F, Wang YY, Jiao L, Dong HL, Huang YY, Jiang HC (2012) Microbial diversity in cold seep sediments from the northern South China Sea. Geosci Front 3:301-316
Zhu D, Tanabe SH, Yang C, Zhang W, Sun J (2013) Bacterial community composition of South China Sea sediments through pyrosequencing-based analysis of $16 \mathrm{~S}$ rRNA genes. PLoS One 8: e78501

Publisher's note Springer Nature remains neutral with regard to jurisdictional claims in published maps and institutional affiliations. 